le double du couple de démarrage. Il y aura donc souvent patinage car l'effort obtenu dépassera en général l'adhérence. Les roues se mettront à tourner en sens inverse et la voiture glissera sur les rails, un patinage même momentané diminuant considérablement l'adhérence. Dans ce cas le freinage ne présentera aucune sécurité.

Enfin dans le cas, le plus tréquent, d'un montage de deux moteurs en série, le patinage d'un seul essieu, provenant par exemple d'une inégalité de charge, suffit à rendre inefficace ce mode d'arrêt. En effet, la force contre-électromotrice $e_{1}$ de celui qui patine est élevée et l'on a:

$$
I=\frac{E-e_{1}+e_{2}}{2 R+p}
$$

Comme d'ailleurs $e_{\mathrm{g}}$ est peu élevé, $I$ sera peu intense et le moteur $\mathrm{n}^{0} 2$ ne donnera aussi qu'un faible couple de freinage.

Pour toutes ces raisons l'usage de cette méthode n'est pas à recommander et ne peut guère être justifié que pour un arrêt de secours, la voiture n'ayant aucun autre frein électrique. Nous l'avons vu cependant employer d'une manière courante par certains mécaniciens de tramways qui s'en servaient même en mettant les moteurs sur la ligne sans intercaler de résistances. Aussi, toutes les fois que l'on pourra le faire, nous conseillons avec MM. Blondel et Dubois ( $\mathrm{I}$ ), de supprimer l'inversion de marche sur les tramways oú la manœuvre peut se faire des deux plateformes, tout en mettant à la disposition du mécanicien un freinage électrique d'un emploi plus sûr, la mise cn courtcircuit des moteurs par exemple.

Dans le cas oú il est nécessaire que le mécanicien puisse se servir facilement de la marche arrière, par exemple sur les lignes mixtes à caniveau et à trolley, afin d'arrêter la voiture juste sur la trappe des points terminus du caniveau, il serait désirable que l'on ne pût, tout au moins, dépasser la position de démarrage pour la marche arrière comme on peut en général le faire avec le matériel américain qui permet la marche arrière à toutes les vitesses.

Un cas cependant ou ce procédé pourrait être employé utilement est celui de certains chemins de fer à crémaillère à courant continu. Lorsque l'usine génératrice est hydraulique, on préférera, en général, ne pas utiliser la récupération qui, comme nous l'avons déjà dit, peut occasionner des décharges momentanées trop grandes des génératrices, surtout si le trafic est peu intense. Dans ce cas, le moteursérie est bien préférable au moteur en dérivation et l'on réalisera le freinage à la descente en inversant la marche. Le courant sera maintenu à une intensité convenable par un rhéostat convenablement dimensionné, à liquide de préférence. On pourra ainsi absorber une énergie sensiblement constante, soit à la montée, soit à la descente, et prévenir tout emballement des turbines.

Pour obtenir le même couple à la même vitesse qu'à la montée, le rhéostat $r$ devra satisfaire à la relation :

$$
\frac{E-e}{R}=\frac{E+e}{R+r} ; \quad(E-e)(R+r)=(E+e) R
$$

(1) La traction électrique sur voies ferrées. - A. Blondel et F. P. Dubois. Tome second, page 205. Baudry. d'où : $\quad r=\frac{2 e R}{(E-e)} \quad$ qui exprime que le courant est le même dans les deux cas.

Ce rhéostat sera donc assez volumineux, et c'est pour cela que nous préférons un rhéostat liquide. Il sera d'ailleurs facile à loger sur une locomotive de chemin de fer à crémaillère.

(A suivre)

J. Bethenod,

Ingénieur E. C. L.

\section{TRAITEMENT DES MINERAIS DE CUIVRE AU FOUR ÉLECTRIQUE}

Nous pensons être agréables à nos lecteurs en reproduisant ici la conférence suivante, faite à la Société des Ingénieurs civils, le 19 juin dernier, par $M$. Ch. VAtTIER, sur les expériences de traitement des minerais de cuivre au Four Keller en fonctionnement aux usines de livet (Sociétẻ Electrochimique de La Romanche), dont une partie de l'énergie est, comme on le sait, utilisée aux opérations de la Compagnie Electro-Thermigue Keller, Leleux ét $C^{\prime a}$.

Il y aura bientôt deux ans, j'avais l'honneur, dans cette même salle, de vous parler des remarquables expériences réalisées sur des minerais de fer dans des fours électriques. Je vous décrivais également les usines électro-métallurgiques que j'avais eu l'occasion de visiter aux Etats-Unis et en Europe et vous manifestais alors, prévision qui a été réalisée, mon espoir que bientôt ces nouveaux procédés, rendus si économiques et si pratiques par les ressources de la houille blanche, quitteraient les domaines du laboratoire et des petites usines expérimentales, pour entrer franchement dans la grande voie industrielle.

Vous connaissez tous l'histoire, encore récente, de l'électro-métallurgie: s'appuyant sur les savantes observations des Moissant, Siemens, Acheson, Minet et autres notabilités de la science, nous avons vu lutter avec acharnement des hommes de science et d'industrie, comme : Stassano, Héroult, Laval, Keller et tant d'autres qu'il serait trop long de mentionner ici, pour créer une nouvelle métallurgie électrique qui est la métallurgie de l'avenir.

Chargé, deux fois en l'espace de trois ans, d'une mission du gouvernement Chilien en Europe et aux Etats-Unis, pour y étudier les nouveaux procédés électro-métallurgiques, je crus utile, en août dernier, d'apporter avec moi du Chill environ $200 t$ de minerais de cuivre, de fer et de manganèse, pour exécuter en France des expériences industrielles de fonte de ces minerais dans des fours électriques; ce sont les résultats de ces expériences qui constituent le but principal de cette conférence, qui sera des plus brèves, en vue de la nécessité de laisser aujourd'hui la parole à plusieurs éminents conférenciers.

Après quelques expériences préliminaires, je fus autorisé à faire de nouvelles études sur le même sujet, dans l'usine de MM. Keller et Leleux, sous la direction de M. Keller, l'usine de Kerrousse, en Bretagne.

C'est là que nous avons arrêté, d'accord avec M. Keller, les forme et marche définitives des fours et appareils accessoires qui, dernièrement et à plusieurs reprises, ont fonctionné, d'une façon parfaite, à la grande usine électro-métallurgique de MM. Keller et Leleux, à Livet, près Grenoble. 
Une première série d'expériences, réalisées dans des proportions praiment industrielles et dans des fours tels que ceux qui seront adoptés dans les futures usines, nous avait prouvé que, pour les minerais de cuivre, le problème était complètement résolu.

Le $2 \mathrm{I}$ avril dernier, nous avons répété à Livet ces mêmes expériences (I) sur diverses espèces de minerais de cuivre, provenant du Chili, devant une Commission composée de:

M. STEAD, ingénieur métallurgiste à Middlesbrough ; M. Atren, ingénieur métallurgiste à Sheffield; M. ReynOLD's, ingénieur métallurgiste à Londres; M. PIRIE, député d'Aberdeen au Parlement Britannique; M. Saladin, ingé- nieur en chef du Creusot; M. De la Bovglise, ingénieur des Mines, Paris ; M. Renevey, ingénieur, Paris; M. Bougère,

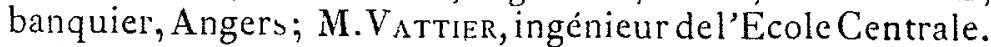

Un procès-verbal, signé, de ces expériences accompagne cette note. M. Keller dirigeait la partie électrique des expériences et le soussigné la partie métallurgique.

Avant d'aller plus loin, je dois d'abord préciser le but que nous voulions atteindre:

Il ne s'agit pas, ici, d'une pierre philosophale qui transforme tout à coup du minerai de cuivre en cuivre métallique pur, ni qui vienne bouleverser les réactions bien connues de la vieille métallurgie de cuivre.

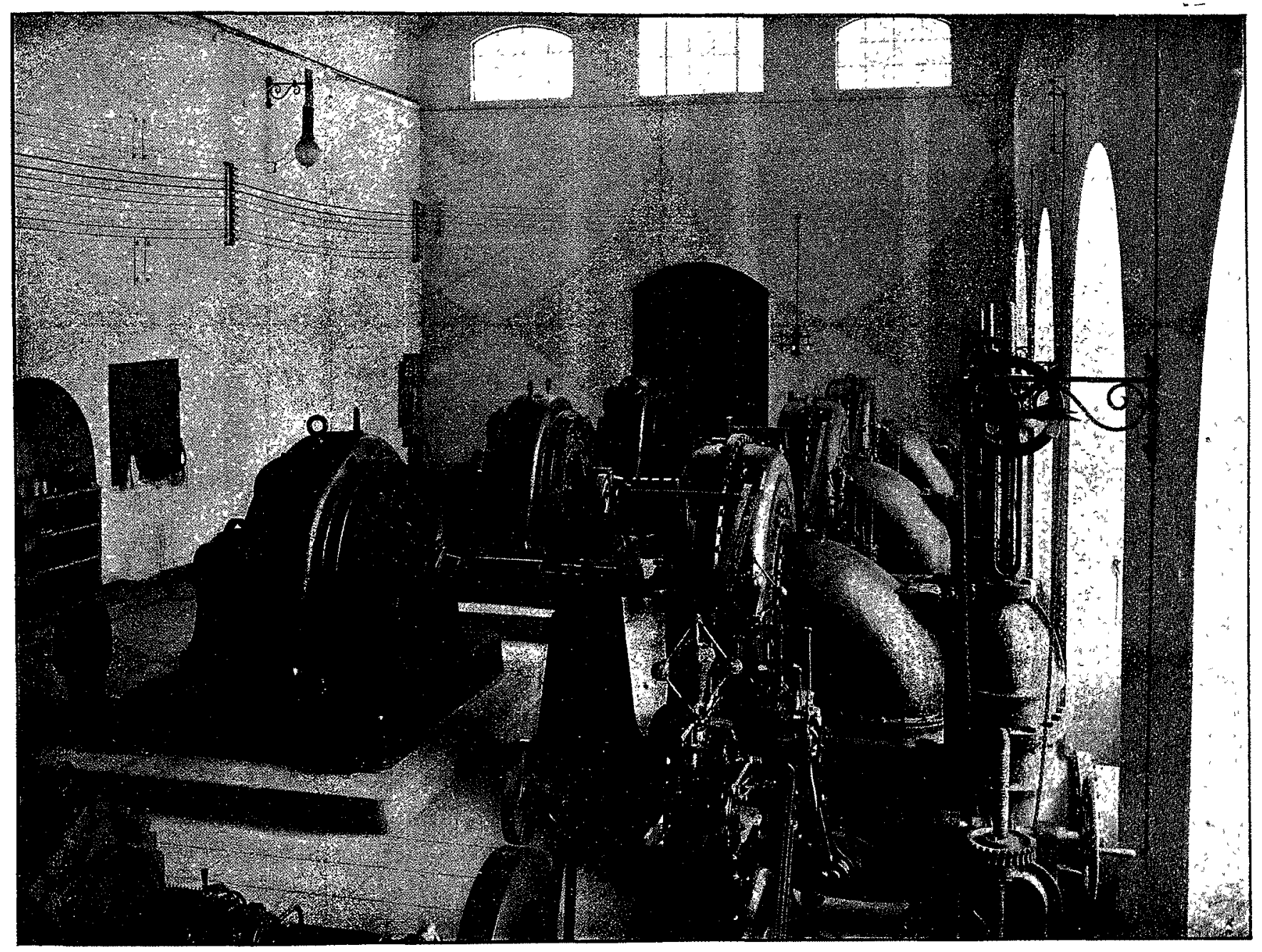

Salle des machines de la Compagnie Keller-Leleux, à Livet.

(1) Cette expérience a été effectuée à l'usine de la Compagnie Electro-thermique Keller, L.eleux et Clè à Livét, le 21 avril igo3, en présence de :

MM. Stead, ingénieur métallurgiste, à Middlesbrough; Allen, ingénieur métallurgiste, à Sheffield ; ReYNol.o's, ingénieur mètallurgiste, à Londres ; Pirie, député d'Aberdeen au Parlement Britannique; Saladin, ingénieur en chef du Creusot; VatTIER, ingénieur chargé de mission par le gouvernement du Chili ; DE LA Bovglise, ingénieur des Mines, Paris; Renevey, ingénieur à Paris; Bovgère, banquier à Angers.

Les expériences ont été dirigées par M. A. Keller, ingénieur, directeur technique de la Compagnie Electro-thermique Keller, Leleux et Cie.

Le traitement a été effectué avec le four électrique double, système Keller.

Minerais. - Composé de minerais : du Volcan, Magnère, Charlin, en proportions indiquées par M. Vattier.
Il a été fondu $8.000 \mathrm{~kg}$ de mélange en huit heures de fonctionnement. Puissance. - Elle a été de : 4.750 (ampères) $\times 119$ (volts) $\times(0,9$ $\cos \varphi)=500$ kilowatts.

Fonctionnement. - Tout à fait normal.

Consommation d'électrodes. - Les électrodes employées étaient de mauvaise qualité ; malgré cela, l'usure relevée a ete en longueur de 6 à $7 \mathrm{~kg}$ par tonne de minerai.

M. Keller fait remarquer que, dans des expériences précédentes, avec une qualité un peu meilleure, il avait obtenu une consommation de $5 \mathrm{~kg}$ maximum par tonne de mineral, et qu'avec l'emploi d'ćlectrodes en graphite, la consommation serait plus réduite encore.

Produits obtents. - La matte et les scories ont été coulées séparément; les analyses ont été faites et ont donné : mattes, $\mathrm{Cu}=43 \mathrm{o}$; scories, $\mathrm{Cu}=0, \mathrm{i}$ o/0.

Signé : C. Vattier, A. Allen, E. Stead, A. Reynold's, Renevey, D.-V. Pirie, de la Bouglise, G. Bougtike, E. Keller. 
Il ne s’agit pas non plus d’un procédé qui supprime toutes les dépenses habituelles et arrive à faire gagner des millions... imaginaires, avec n'importe quel minerai et dans n'importe quelle région!

Laissons à des prospectus fantaisistes, qui circulent en ce moment dans certains centres financiers, le monopole de ces élucubrations, qui coûteront cher aux naîfs, et prenons cette question de plus haut, à un point de vue scientifique et vraiment industriel, et surtout à un point de vue qui permette ì tous les vrais travailleurs de perfectionner encore ces procédés et d'en tirer de légitimes bénéfices.

Tout le monde sait que, généralement, dans la métallurgie du.cuivre par la voie sèche, soit dans des fours à réverbère, soit dảns des fours à manche, on transforme d'abord, avec de la houille ou du coke, les minerais de cuivre en mattes de cuivre d'un titre variable entre 40 et $50 \%$, et qu'ensuite ces mattes sont soumises, soit à des grillages avec refonte, soit au traitement spécial des convertisseurs ou sélecteurs, ou aux fours à réacteur Thofern et Saint-Seine, etc., pour être transformées en barres de cuivre que l'on raffine dans des fours, qu'on soumet à l'électrolyse, pour en retirer du cuivre électrolytique et en séparer l'or et l'argent.

Pour le moment, nutre but est uniquement de supprimer la dépense de la houllle noire (coke, houille ou charbon de bois) qu'on emploie pour la fonte du minerai jusqu'à la matte, et de remplacer les calories produites par la houille noire par des calories électriques produites par la houllle blanche, c'est-à-dire par des forces hydrauliques.

Une fois cette matte obtenue, nous la livrons aux appareils actuellement employés en métallurgie (convertisseurs, sélecteurs, réacteurs, etc.), et c'est tout au plus si nous demandons à l'électricité de nous prêter śon concours pour achever les réactions, là où des électrodes peuvent encore nous fournir les calories nécessaires.

En un mot, nous cherchons surtout à appliquer ces procédés là où le charbon est cher et où on peut se procurer, dans de bonnes conditions économiques, de puissantes forces hydrauliques, et c'est la différence entre le coût de la houille noire et celui de la houille blanche, qui constituera le principal bénéfice que feront réaliser ces nouveaux procédés C'est dans cet ordre d'idées que nous avons réalisé ces dernières expériences dont je vais vous faire une succinte description.

Minerais. - Nous avons opéré sur deux classes de minerais :

Io Minerais de cuivre provenant de la mine du «Volcan» (Chili), de M. Gregorio Donoso.

Le titre en cuivre était d'environ $7 \%$, sous forme de sulfure jaune de cuivre.

Ce minerai contient de 8 à $9 \%$ de soufre et sa gangue se compose de silicates, silice, un peu de carbonate de chaux et surtout d'oxyde de cuivre micacé.

$2^{0}$ Minerais de cuivre très pauvres provenant des centres miniers voisins de Santiago (Chili), mélangés avec un peu d'oxyde de manganèse (du Chili) et de chaux.

La composition du lit de fusion était la suivante :

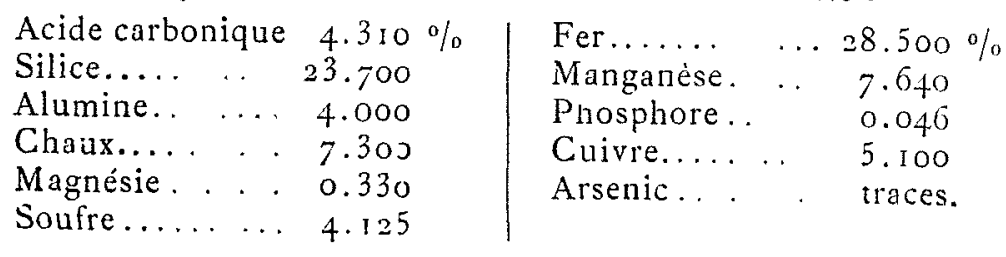

Ces minerais étaient, partie en morceaux assez volumineux, et partie en poudre.

On chargeait indifféremment à la pelle dans le creuset, les morceaux, comme les menus et les poussières, sans avoir à éprouver les inconvénients qui résultent de la présence des fins dans les fours à manche. Toutes les charges passées étaient soigneusement pesées et échantillonnées et on tenait un compte précis de la durée des diverses phases des expériences.

Des blocs assez volumineux et réfractaires, après un mouvement giratoire prononcé, ne tardaient pas à être dissous dans le lit de fusion.

Fours. - Le four proprement dit, de première fusion ou de dislocation des molécules par la chaleur, se compose d'un creuset rectangulaire en briques réfractaires dont les dimensions sont :

Longueur $=1,800 \mathrm{~m} ;$ largeur $=0,90 \mathrm{~m}$; hauteur $=0,90 \mathrm{~m}$.

En dessous de ce creuset se trouve l'av'ant-creuset destiné à permettre la séparation bien nette de la matte et de la scorje; cet avant-creuset a comme dimensions :

Longueur $=\mathrm{I}, 20 \mathrm{~m}$; largeur $=0,60 \mathrm{~m}$; hauteur $=0,60 \mathrm{~m}$.

Des orifices, qu'on peut boucher à volonté par de petits tampons d'argile, sont ménagés au fond du creuset supérieur. dont les produits s'écoulent à volonté par un petit canal dans l'ayant-creuset inféricur.

Dans le creuset supérieur plongent à volonté, ou sont amenés au ras du bain, deux électrodes en charbon d'une section carrée de $0,30 \mathrm{~m}$ de côté et $1,70 \mathrm{~m}$ de longueur.

Dans l'avant-creuset pénctent deux électrodes réchauffeurs de $0,25 \mathrm{~m}$ de côtć.

Des orifices ménagés à divers niveaux de l'avant-creuset et débouchés au moyen "d'un fleuret en acier, muni d'un bouton mobile pour recevoir le choc du marteau, permettent à volonté de fairé écouler, soit les scories dans des rigoles en sable, soit les mattes dans des lingotières en acier, mues par un trétil roulant sur un pont situé à la partie supérieure de l'atelier. Des .voltmètres et ampèremètres permettent de régler et préciser les intensités des courants.

On a marché avec courant alteriatif.

Une combinaison spéciale permet à volonté d'élever et d'abaisser facilement et séparément chacun des électrodes.

Marche des opérations. - On descend les deux grands électrodes dans le creuset supérieur et on établit le courant en mettant quelques charbons et petits -morceaux de matte au fond de ce creuset, dont on élève graduellement la température.

Les minerais sont élevés, par un treuil, sur une plateforme supérieure et sont chargés autour des électrodes, lesquels sont élevés à mesure que la charge augmente.

La fusion commence rapidement et, quand le creuset est plein de matières fondues ou semi-fondues, on déboucheau fleuret un orifice situé à environ $0,10 \mathrm{~m}$ du fond du creuset.

Le liquide, plus ou moins pâteux (au bout de quelque temps très fluide), s'écoule dan's l'avant-creuset où les réactions s'achèvent, et on facilite la fin de ces réactions et la séparation des liquides au moyen des électrodes réchauffeurs. 
Quand cet avant-creuset est à peu près plein, on fait écouler les scories par un des orifices supérieurs, et, quand on voit que la matte occupe un espace suffisant, on fait écouler cette matte par un orifice inféricur. On procède ainsi par cuvées successives, tant dans le creuset supérieur que dans l'avant-creuset.

USINE DE LA COMPAGNIE ÉLECTROTHERMIQUE KELLER-LELEUX, A LIVET

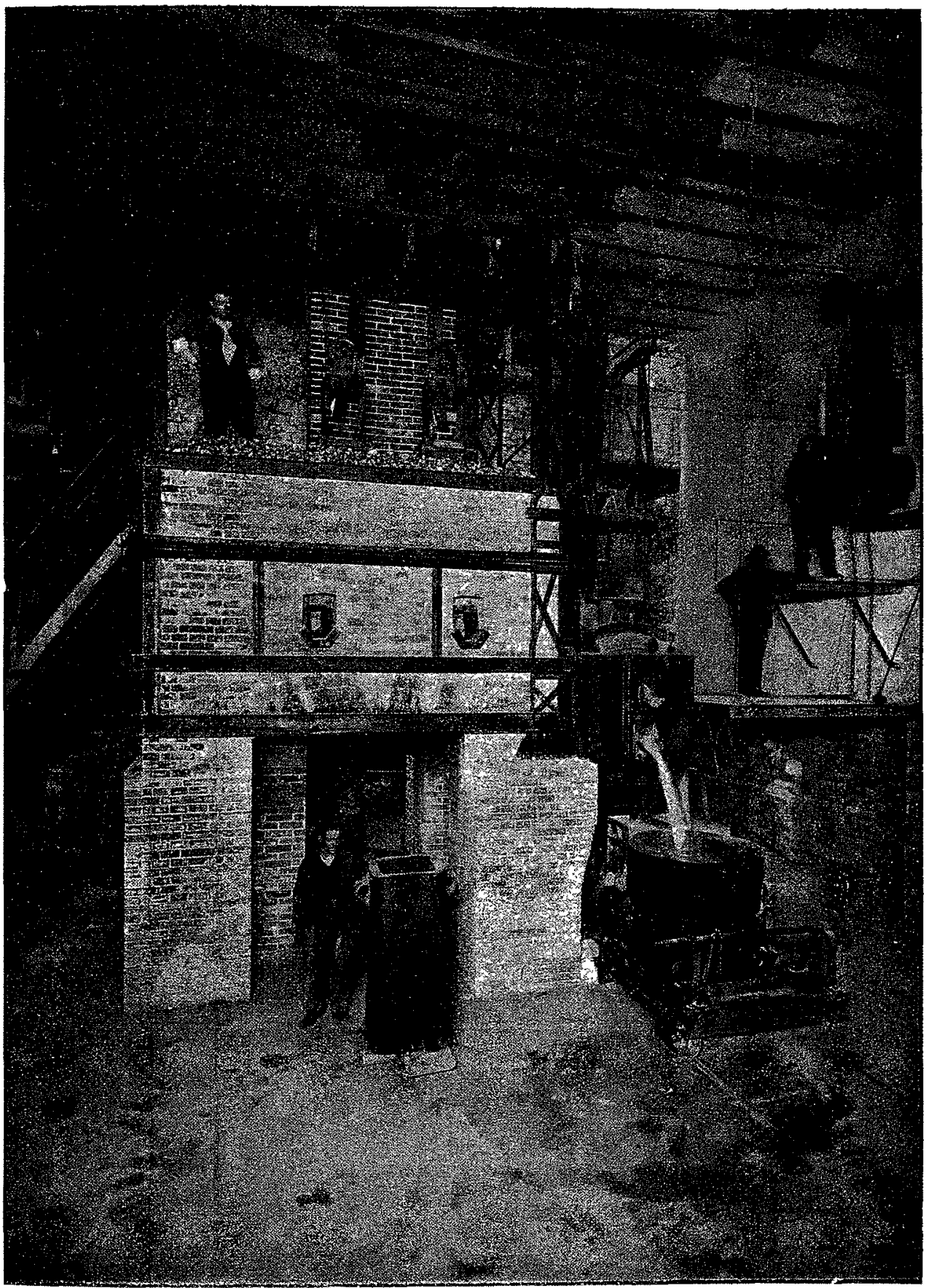

Four électrique double de roo HP (Procédé Keller). de $76 \mathrm{kgm}$. l'analyse a donné :

Ainsi, par exemple, pour fondre roo $t$ de minerais par vingt-quatre heures, il faudra disposer, aux dynamos, d'une force de 2.833 chevaux, mettons : 3.000 cheraux

Produrts obtenus. - On a recucilli des mattes dont

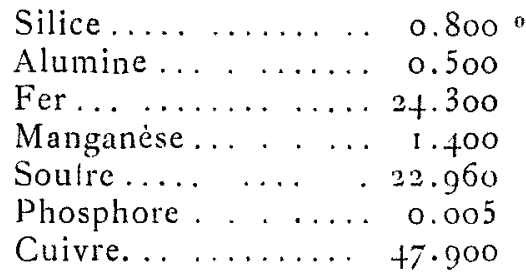

et des scories de la composition suivante :

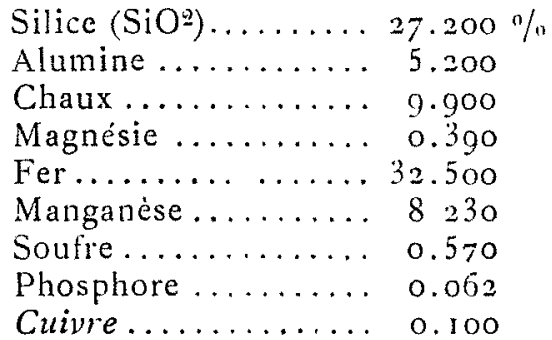

Les premières et dernières scories donnent généralement un titre un peu plus élevé en cuivre.

On pourrait aussi tirer un bon parti des scories, à cause de leurs titres en fer et silice, pour fabriquer du ferro-silicium, en faisant directement écouler ces scories dans un creuset spécial et les soumettant de suile, afin de profiter de leurs calories acquises, à une haute température dans un four électrique spécial.

Ces mêmes scories, chargées d'éléments manganésifères, pourraient ainsi ètre aussitôt transformées en ferro-manganèses et spiegels.

Obsrrvations. - Pendant la marche des opérations, il convient d'arriver, au moyen d'un voltage suffisant, à faire pisser l'arc ou courant électrique entre les deux électrodes, cn maintenant ces derniers au ras du bain, afin d'éviter autant que possible leur contact avec ce bain. En eflet, le charbon, à ces hautes températures, tend à réduire l'oxyde de fer en fer métallique, ce qui donne les divers inconvénients suivants :

Eléments de la marche it de la production. - Avec ce four on peut fondre pour matles, environ $25 t$ de minerais par vingt-quatre heures.

On a marché avec : 4.750 ampères; II9 volts; $\cos \%$ $=0,9$, ce qui correspond à 500 kilowatts ou 680 chevaux de force.
1. Usure plus rapide des électrodes;

$2^{\circ}$ Dépense d'énergie électrique supplémentaire et inutile; $3^{0}$ Abaissement du titre en cuivre des mattes.

Avec des électrodes en graphite d'Acheson, cet inconvénient sera moindre.

Mais, sans chercher absolument aucune amélioration à 
la situation actuelle du problème, si nettement résolu à Livet, on peut déjà se rendre parfaitement compte des avantages économiques et autres de ce nouveau procédé électro-métallurgique.

\section{Comparaison entre les anciens procédés métallurgiques} du cuivre et ceux de l'électro-métallurgie. - Prenons le cas d'une mine de cuivre située dans l'Amérique du Sud, au Chili par exemple, à une assez grande distance de la côte, au pied de la Cordillère, comme le a Volcan » (d'où proviennent les mmerais soumis aux expériences), c'està-dire là où le coke vaut au moins ıoo $f$ la tonne et où, à cause de la pente du terrain et de la situation locale, on peut se procurer une puissante force hydraulique, constante et économique.

Rapportons les chiffres comparatifs à une tonne de cuivre en barres qu'on retirera de ces minerais du «Volcan » d'un titre en cuivre d'environ $7 \%$, titre relativement assez élevé et qui, on doit le reconnaître, est supérieur à la moyenne de ce qu'il faut compter pour des exploitations sur une grande échelle.

Pour produire une tonne de cuivre, il faudra fondre environ $16 t$ de ces minerais; et laissant les mêmes facteurs relatifs à l'exploitation du minerai et transformation des mattes, étudions les éléments comparatifs des deux solutions :

Io Dans les fours à manche actuels au coke, pour produire cette tonne de cuivre dans des mattes, il faudra $3.200 \mathrm{~kg}$ de coke à $100 \mathrm{f}$ la tonne, c'est-à-dire une dépense de $320 \mathrm{f}$;

$2^{\circ}$ Dans les fours électriques, la fonte de $16 t$ de minerais exigera une énergie de I, 25 kilowatt-an, et, comme il faut compter, dans cette région, le kilowatt an à raison de $30 \mathrm{f}$, la dépense d'énergie sera de 1,25 kilowatt $\times 30=37,50 f$, mettons: $38 \mathrm{f}$. La dépense en électrodes constatée par l'expérience a été de $75 \mathrm{~kg}$ par tonne de cuivre contenue dans les mattes (cette dépense peut être de beaucoup réduite par le dispositif des électrodes dont j'ai parlé et surtout par l'emploi d'électrodes de graphite), de sorte qu'il faut compter sur une dépense actuelle de $45 f$ pour cette usure d'électrodes.

En un mot, le procédé électro-métallurgique pour la fonte des $16 t$ de minerais introduira une dépense de:

$$
38+45=83 f
$$

et l'économie sur le procédé des fours à manche, pour ces facteurs principaux des calories, sera, par tonne de cuivre:

$$
320-83=237 f \text {, ou plus de } \& 9 \text {. }
$$

Les autres avantages introduits par l'emploi des fours électriques, de grande importance au point de vue pratique, sont les suivants:

$1^{0}$ Suppression des ventilateurs, indispensables dans les fours à manche;

$2^{0}$ Possibilité, avec l'électricité, de fondre des lits de fusion beaucoup plus réfractaires que ceux passés aux fours à manche;

3o Suppression de la confection des briquettes ou agglomération des menus et poussières;

$4^{\circ}$ Réduction de la main-d'œuvre;

50 Suppression des accrochages, si fréquents et si coûteux qui se produisent dans les fours à manche et suppression, également, des engorgements des creusets.
Maintenant, si nous prenons des minerais de cuivre de $4 \%$, nous voyons que, pour concentrer une tonne de cuivre dans les mattes, il faudra:

Io Dans les fours à manche, une dépense supplémentaire en coke de $: \frac{100}{1.000} \times(5.1000-3.200)=180 f$

$2^{\circ}$ Dans les fours électriques, au lieu de I, 25 kilowatt-an, il faudra 2 kilowatts-an, ou simplement une dépense supplémentaire de :

$3 \circ f \times(2$ kilowatts-an $-1,25$ kilowatts-an $)=22,5 \circ f$, c'est-à-dire une économie supplémentaire de I $57,50 \mathrm{f}$, chiffre relativement considérable pour un minerai de bas titre.

Naturellement, il est impossible de fixer un chiffre moyen représentant l'économie qu'on réalisera pour chaque tonne de cuivre contenue dans des mattes... Cela dépendra des titres des minerais et surtout des conditions locales, mais je ne crois pas exagéré le chiffre probable de $£$ Io, comme moyenne de l'économie réalisée par tonne de cuivre pour le traitement des minerais de cuivre de l'Amérique du Sud, dans des mines situées loin de la côte et à proximité de puissantes et constantes chutes hydrauliques.

Conclusion. - Il convient à nos métallurgistes de continuer des études pratiques sur les applications des fours électriques, non seulement à la fonte des minerais de cuivre, mais au traitement d'autres minerais et, principalement, à celui des blendes argentifères, très abondantes dans certaines régions (en Bolivie, par exemple) et que, jusqu'ici, on n'a pu traiter d'une façon économique.

Je ferai la même observation pourles minerais de fer etde manganèse, qui, dans les régions lointaines, attendent une solution pratique permettant de les utiliser, en évitant des transports trop con̂teux, avec les bas prix actuels de ces métaux.

Malgré les avantages évidents de ces nouveaux procédés électro-métallurgiques, lesquels sont certainement appelés à un grand avenir dans certains pays, comme le Chili, par exemple, où les conditions industrielles sont si favorables a tous les points de vue et surtout aux points de vue mines et chutes d'eau, il est de mon devoir, néanmoins, de mettre nos collègues et les capitalistes en garde contre les exagérations, qui peuvent avoir de funestes conséquences.

Avant de risquer le moindre capital dans une entreprise électro-métallurgique dans les régions lointaines, il faudra s'assurer avec le plus grand soin:

Io $\mathrm{Si}$ nn peut compter, non sur le papier, mais bien réellement et avec des titres de propriété en règle, sur des mines pouvant livrer des quantités de minerais de titre suffisant pour motiver des installations électro-métallurgiques, lesquelles sont très coûteuses à cause de la création de puissantes forces motrices hydrauliques.

$2^{0} \mathrm{Si}$, près de ces mines, ou au moins à des distances qui permettent des transports de forces par l'électricité pas trop onéreuses, on peut acquérir d'une façon certaine et à l'abri de tout procès ou toute réclamation, de très puissantes et surtout constantes forces hydrauliques;

3. Si on trouvera, dans la région choisie pour les installations, la main-d'cuvre suffisante pour l'exploitation des mines, et des conditions industrielles permettant les installations.

Une longue expérience m'a démontré que ces conditions, 
possibles à réaliser sur quelques points privilégiés, sont généralement bien rares, et il faut défendre notre épargne française contre le mirage des spéculations plus ou moins risquées et fantaisistes qui la menacent.

Soyons heureux, enfin, de constater que c'est en France qu'on a réalisé, avec le plus de succès, les progrès de l'électro-métallurgie et, qu'une fois de plus, nos compatriotes sont à la tête d'un mouvement scientifique et industriel.

\section{Ch. VAtTIER,}

Ingénieutr de l'Ecole centrale.
Plaçons-nous dans la situation d'un industriel qui veut aménager une force hydro-électrique sur une rivière donnée. Nous allons choisir sur cette rivière, la section dont la longueur nous founira la plus grande hauteur de chute. Cette longueur et cette hauteur sont, en pratique, pour une puissance déterminée à obtenir, des variables liées entre elles par des fonctions très complexes et dont les principales sont : l'acquisition des droits de riveraineté, les servitudes préexistantes, la difficulté des travaux de dérivation et le choix de l'emplacement de l'usine. Toutes ces considérations ayant été bien appréciées, au moyen d'études préliminaires, nous sommes conduits à fixer la hauteur de chute à un chiffre de... mètres. Il s'agit maintenant de

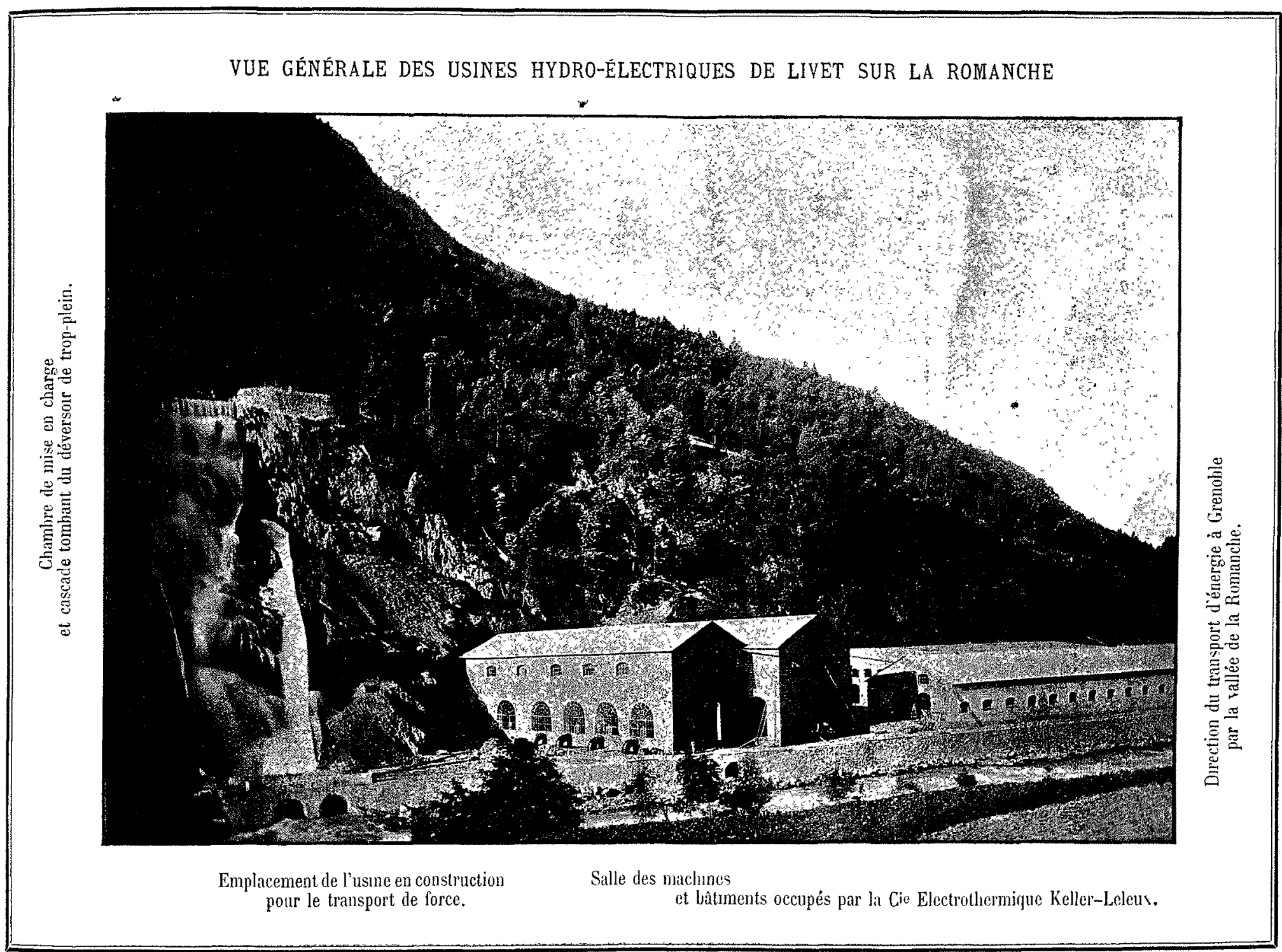

\section{TRANSPORT DE FORCE ET ÉLECTROCHIMIE}

L'article qu'on vient de lire m'amène à citer les installations de la "Société Electrochimique de la Romanche "comme le premier exemple de réalisation en grand de ce qu'on pourrait appeler le principe de la meilleure utilisation des forces bydroélectriques, admis par tous les économistes en matière de houille blanche, fondement des plus beaux projets d'avenir de cette industrie, qui sera celle du $x^{e}$ siècle.

Je tiens à m'expliquer tout de suite sur ce que j'entends par "principe de la meilleure utilisation" pour bien montrer que Ie n'ai pas, hors de propos, l'intention de rien innover, mais seulement le désir de relier les unes aux autres, par des déductions logiques, des théories concordantes et qui pourtant n'apparaissent pas telles, parce qu'émises différemment selon les auteurs. savoir pour quel débit nous devons construire les ouvrages de dérivation.

Considérons la courbe de régime (1) de cette rivière, soumise à l'influence des glaciers et des neiges. Son débit caractéristique d'etiage (2), pour employer les définitions très commodes de $M$. Tavernier, est, je suppose, de 6 mètres cubes, et son débil caractéristique moyen (3) de 20 mètres cubes. Pour quel débit entre ces deux limites aménagerons-nous une chute sur

(1) Je suppose que le jaugeage du plus grand nombre de nos rivières industriellement utilısables ait été fait, ce qui arrivera bien un jour, et qu'on n'ait plus qu'à consulter des atlas de graphiques. Inutule de faire ressortir tout l'intérêt de ce travail.

(2) Au-dessous duquel il descend pendant dix jours par an au plus, en année sèche.

(3) Au-dessous duquel il descend pendant 180 jours au plus, en année morenne (moyenne de ro années consécutives). 